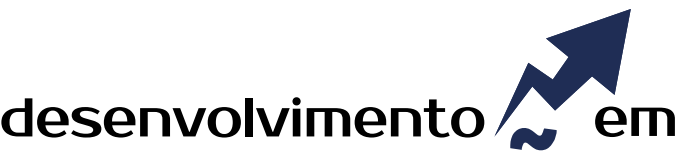 QUESTÂO
}

\section{Da Crise à Oportunidade: \\ Uma Análise da Orientação Empreendedora de Brasileiros nos EUA}

http://dx.doi.org/10.21527/2237-6453.2019.46.45-55

Recebido em: $11 / 7 / 2016$

Aceito em: 23/4/2018

\author{
Daniel Penz ${ }^{1}$, Bianca Costa Amorim ${ }^{2}$, Elisabete Penz Beuren ${ }^{3}$ \\ Sabrina do Nascimento ${ }^{4}$, Carlos Ricardo Rossetto ${ }^{5}$
}

\begin{abstract}
RESUMO
Diante da possiblidade de um novo negócio o empreendedor depara-se com as dificuldades do ambiente e com a deterioração das condições econômicas do Brasil. Tais fatores têm estimulado as pessoas a imigrarem, levando consigo o sonho de empreender. Este artigo teve como objetivo geral identificar o grau de Orientação Empreendedora (OE) existente nos brasileiros que optaram por investir seus esforços e iniciaram novos empreendimentos nos EUA. Para alcançar este objetivo fez-se uma pesquisa quantitativa, com o uso de modelagem de equações estruturais, utilizando o software SmartPLS, com o uso de dados primários. A população desta pesquisa é composta por 3.657 empresários brasileiros estabelecidos nos Estados Unidos que estão cadastrados na Associação Brasileira de Empreendedores nos Estados Unidos; a amostra compreende 107 empreendedores. Para gerar os resultados estimou-se o modelo estrutural no software SmartPLS, com todas as questões originais de cada construto; na sequência avaliou-se quais possuíram um bom ajuste, verificou-se a validade discriminante e a confiabilidade. Vencidas estas etapas foram verificados os coeficientes estruturais e $R^{2}$ para chegar no modelo final. Identificou-se a partir dos dados da pesquisa que a amostra estudada possui as características propostas por Miller (1983) nas seguintes proporções: $56 \%$ para inovação, $78 \%$ para proatividade e $73 \%$ para tomada de risco. Esses valores caracterizam os brasileiros que imigraram para os EUA com um comportamento altamente proativo, comprometendo-se fortemente com a tomada de risco, apostando medianamente nas inovações.
\end{abstract}

Palavras-chave: Orientação empreendedora. Novos negócios.

FROM THE CRISIS TO THE OPPORTUNITY: AN ANALYSIS OF ENTREPRENEURIAL ORIENTATION FROM BRAZILIAN IN USA

\begin{abstract}
Glimpsing the possibility of a new business the entrepreneur is faced with the difficulties of the environment and the deterioration of economic conditions in Brazil, these aspects have encouraged people to immigrate, and had the dream of taking. This article aimed to identify the degree of Entrepreneurial Orientation (OE) existing in Brazilians who opted to invest their efforts and started new ventures in the USA. To achieve this goal, a quantitative research was done using the structural equation modeling using the SmartPLS software, using primary data. The population of this research is composed of 3,657 Brazilian businessmen established in the United States who are registered in the Brazilian Association of Entrepreneurs in the United States, the sample comprises 107 entrepreneurs. In order to generate the results, we estimated the structural model in the SmartPLS software, with all the original questions of each construct, in the sequence, we evaluated which ones had a good fit, verified the discriminant validity and the reliability. the structural coefficients were verified and R2 to arrive at the final model. It was identified from the research data that the sample studied has the characteristics proposed by Miller (1983) in the following proportions: $56 \%$ for innovation, $78 \%$ for proactivity and $73 \%$ for risk-taking. These values characterize Brazilians who have immigrated to the United States with a highly proactive behavior, are strongly committed to risk taking and are fairly committed to innovations.
\end{abstract}

Keywords: Entrepreneurial orientation. New business.

\footnotetext{
${ }^{1}$ Mestre em Administração pela Universidade do Vale do Itajaí. Pesquisador da Fundação Certi e professor da faculdade Cesusc. penz.daniel@gmail.com

2 Doutoranda em Administração pela Universidade do Estado de Santa Catarina - Udesc. Mestre em Administração pela Universidade do Vale do Itajaí. Professora do Centro Universitário Leonardo da Vinci. bianca.costa.amorim@gmail.com

${ }^{3}$ Mestre em Ensino pela Universidade do Vale do Taquari. Professora de Ensino Fundamental e Médio da Sociedade Educação e Caridade. flavioebetti@brturbo.com.br

${ }^{4}$ Doutora em Administração pela Universidade do Vale do Itajaí. Professora titular da Universidade do Oeste de Santa Catarina. sabnascimento@gmail.com

${ }^{5}$ Doutor em Engenharia de Produção pela Universidade Federal de Santa Catarina. Professor da Universidade do Vale do Itajaí. rossetto@univali.br
} 
O processo de globalização fomenta o aumento da velocidade com que as indústrias e os ciclos de vida ocorrem (FILLIS, 2001), possibilitando o ingresso de muitas pessoas no mercado como empreendedoras. De acordo com a pesquisa Global Entrepreneurship Monitor - GEM (2016), os empreendedores brasileiros são motivados a abrir um novo negócio por questões de oportunidade e necessidade. O processo de se tornar empreendedor mostra-se uma forma de inclusão social que melhora a distribuição de renda da população, de acordo com Ninis (2013). Vislumbrando a possibilidade de um novo negócio o empreendedor depara-se com as dificuldades do ambiente. No entendimento de Margolis (1995) a deterioração das condições econômicas do Brasil tem estimulado as pessoas a imigrarem, levando consigo o sonho de empreender. Os brasileiros têm escolhido destinos como Nova York, Boston, Miami, São Francisco, entre outras cidades dos EUA. Os imigrantes brasileiros optam por executar trabalhos menos complexos, em nichos de mercados específicos. Ainda que muitos possuam qualificação para diferentes funções, os valores salariais para as funções mais simples nos Estados Unidos são superiores às tarefas mais complexas realizadas no Brasil (MARGOLIS, 1995).

Os empreendedores que imigraram iniciam uma nova empresa para alavancar seu desenvolvimento econômico, gerando o autoemprego (SANDERS; NEE, 1996). Os autores ainda destacam que apesar de o capital humano estrangeiro não ser tão valorizado quanto o local, o mercado de trabalho os acolhe e possibilita a geração de um novo negócio. Para Gibson, Harris e Sadighian (2011), a natureza e o nível do comportamento empreendedor são determinados por uma combinação de características de grupo e oportunidades disponíveis em determinados segmentos de mercado.

O movimento da globalização fornece oportunidades para as empresas se desenvolverem independente de seu tamanho. Segundo Fillis (2001), as pequenas empresas, apesar de seus recursos limitados, possuem a flexibilidade e rapidez na adaptação, o que pode se tornar uma vantagem perante seus concorrentes. A participação das pequenas empresas no mercado apresenta-se como um importante propulsor do desenvolvimento econômico de um país (WIKLUND; SHEPHERD, 2005). Os autores apontam que esta temática ainda necessita ser mais bem estudada e sugerem que são necessárias mais pesquisas acerca da Orientação Empreendedora nas pequenas empresas.

Estudos sobre orientação empreendedora ganharam força. Wiklund e Shepherd (2008) identificam uma carência de estudos nas especificidades, como investigar a forma como os empreendedores adentram nos mercados. Os autores sugerem mais estudos nos tipos específicos de empreendedores ou níveis de análise e variáveis para auxiliar na melhor compreensão da OE.

Miller (2011) destaca a questão do contexto estudado, sugerindo que este muda completamente a abordagem utilizada para analisar e compreender o fenômeno da OE. De acordo com Miller (2011), quanto mais refinado o contexto mais apropriada é a informação extraída e mais valiosa ela é para descrever fenômeno analisado, pois estudos em contextos específicos podem ser muito importantes para práticos e estudiosos.

Orientado nestas premissas este trabalho propõe identificar os índices de Orientação Empreendedora em uma população de pequenos empresários brasileiros que empreenderam nos EUA. A partir das necessidades apontadas chega-se à pergunta de 
pesquisa que servirá de orientação para deste estudo: Qual o grau de Orientação Empreendedora $(O E)$ existente nos pequenos empresários brasileiros que empreenderam nos EUA?

Com o objetivo geral espera-se identificar o grau de Orientação Empreendedora (OE) existente nos pequenos empresários brasileiros que atuam nos EUA. Para atingir o objetivo geral foram estabelecidos três objetivos específicos: a) identificar o grau de inovação na amostra pesquisada; b) caracterizar o grau de proatividade destes empresários e c) apontar o grau em que os empresários estão dispostos a assumir risco.

Este artigo está estruturado em cinco seções. A primeira é a seção introdutória, seguida da fundamentação teórica que servirá de suporte para este estudo; os procedimentos metodológicos utilizados para a consecução desta pesquisa são apresentados na sequência. A quarta seção refere-se à análise e discussão dos dados e como última seção têm-se as considerações finais e sugestões para futuras pesquisas.

\section{ORIENTAÇÃO EMPREENDEDORA}

Uma empresa empreendedora é aquela que se engaja em um produto ou mercado inovador, comprometendo-se com o risco do empreendimento, sendo a primeira a apresentar inovações e ainda com atitude proativa, concorrendo de maneira acirrada com seus concorrentes (MILLER, 1983). Organizações empreendedoras utilizam recursos para o desenvolvimento de projetos cujo resultado é desconhecido. Miller (1983) em seus estudos retirou o foco de análise do indivíduo "empreendedor" e o recolocou no âmbito organizacional, surgindo assim o papel da Orientação Empreendedora (OE) nas empresas.

O fenômeno da Orientação Empreendedora nas últimas décadas tornou-se o foco da literatura empresarial voltada às atividades empreendedoras (COVIN; WALES, 2011). No âmbito da gestão estratégica e do empreendedorismo a perspectiva da Orientação Empreendedora é tratada como um tema central de diversos pesquisadores da área de negócios (SLEVIN; TERJESEN, 2011; SUNDQVIST; KUIVALAINEN; CADOGAN, 2011; SOININEN et al., 2012).

Miller (1983) destaca a OE como um construto unidimensional, e compondo tal construto ele apresenta as dimensões inovação, tomada de risco e proatividade. Merz e Sauber (1995) definem a OE como o grau de proatividade que a organização possui em sua unidade de produto-mercado associado com a sua vontade de inovar e criar novas ofertas.

Lumpkin e Dess (1996) afirmam que a OE se caracteriza como um constructo multidimensional que compreende autonomia, inovação, tomada de risco, agressividade e proatividade. Voss, Voss e Moorman (2005) asseveram que a OE compreende o nível de envolvimento da empresa com seu comportamento, com a tomada de riscos, inovação, proatividade, autonomia e agressividade competitiva que levam as alterações na organização ou no mercado em que esta atua.

Na visão de Avlonitis e Salavou (2007) a Orientação Empreendedora é um fenômeno organizacional que reflete a capacidade gerencial pelo qual as empresas embarcam em iniciativas proativas e agressivas para alterar de maneira vantajosa o ce- 
nário competitivo. Pearce, Fritz e Davis (2010) conceituam a OE como um conjunto de comportamentos distintos, mas relacionados às qualidades de inovação, proatividade, agressividade competitiva, tomada de riscos e autonomia.

A partir das definições de OE encontradas na literatura como as mencionadas por Miller (1983), Lumpkin e Dess (1996), Voss, Voss e Moorman (2005), Avlonitis e Salavou (2007) e Pearce, Fritz e Davis (2010) entre outras, percebe-se que os conceitos apresentados por Miller (1983) e Lumpkin e Dess (1996) configuram-se como constructos que embora distintos são adotados de forma parcial ou integral em vários estudos que abordam a OE.

\section{ORIENTAÇÃO EMPREENDEDORA NA VISÃO DE MILLER (1983)}

A partir do trabalho de Danny Miller em 1983 o tema Orientação Empreendedora ganhou atenção no ambiente acadêmico (WALES; GUPTA; MOUSA, 2011). Miller (1983) destaca em seu trabalho que uma empresa empreendedora é aquela que se engaja em um produto ou mercado inovador, compromete-se com o risco do empreendimento ao ser a primeira a surgir com as inovações e atitudes proativas, batendo forte em seus concorrentes.

As organizações necessitam de renovação contínua de seus processos a fim perpetuar sua sobrevivência num mercado em que a concorrência se apresenta a cada dia mais acirrada. Para Miller (1983), o processo do empreendedorismo e os processos organizacionais são mais importantes do que a figura do empreendedor. Miller (1983) afirma que é por meio dos processos que as organizações se renovam juntamente com os mercados em que atuam. À medida que esta renovação ocorre a Orientação Empreendedora da organização emerge como um conjunto unidimensional que compreende a inovação, tomada de risco e proatividade.

Eggers, Hansen e Davis (2012) destacam que quanto maior o nível das dimensões de inovação, tomada de risco e proatividade na organização, mais o negócio se torna empreendedor. A Orientação Empreendedora aparece quando a organização envolve ações estratégicas, observáveis e especificamente - inovação, proatividade e tomada de riscos - e se essas ações ocorrem com alguma consistência (ANDERSON; ESHIMA, 2013).

A capacidade de inovação de uma organização envolve o uso de uma nova tecnologia em novos produtos ou serviços (MILLER, 1983). Para Schumpeter (1911-1997), o empresário surge como um agente inovador, que traz novos produtos para o mercado oriundos de combinações mais eficientes dos fatores de produção ou se faz valer de uma invenção ou inovação tecnológica. O autor apresenta o conceito de "destruição criadora", que é a substituição de hábitos e produtos antigos por novos. Nessa perspectiva, o produtor é quem inicia a mudança e os consumidores são "ensinados" a desejarem as novidades que acarretam o processo de desenvolvimento econômico (SCHUMPETER, 1911-1997). 
Assumir riscos, no entendimento de Khandwalla (1976), é o grau em que os gestores interagem de forma agressiva com seu ambiente. Para o autor, algumas administrações optam por risco mais elevados buscando maior retorno de investimento, enquanto outros gestores optam por baixo risco e consequentemente baixo retorno dos investimentos.

Miller (1983) entende por tomada de risco a propensão da empresa para se engajar em projetos de risco e as preferências dos gestores por decisões ousados para alcançar os objetivos estratégicos da organização.

Risco é uma condição em que as consequências diretas e indiretas de uma decisão são projetadas (BAIRD; THOMAS, 1985). No entendimento dos autores os gestores estão diante de variáveis que nem sempre são mensuráveis, pode-se afirmar então que eles nem sempre conhecem todos os possíveis resultados, ou as probabilidades de sua ocorrência. Entende-se que a relação do risco com o ambiente incerto em que a empresa está inserida é alvo de concordância na literatura.

Jauch e Glueck (1988) definem o comportamento proativo como vinculado aos atos estratégicos que são empreendidos antes das oportunidades aparecerem ou ainda, antes de serem forçados pelo ambiente a mudarem, ou seja, a empresa estaria se antecipando às demandas do mercado.

Para Lumpkin e Dess (1996) proatividade refere-se à forma como a empresa se relaciona com o mercado, agindo oportunamente a fim de moldar o ambiente, influenciando tendências ou criando demandas. Assim, uma empresa proativa age como um líder ao invés de um seguidor, pois possui vontade e perspicácia para aproveitar as oportunidades.

\section{ORIENTAÇÃO EMPREENDEDORA NA VISÃO DE LUMPKIN E DESS} (1996)

Tomando como ponto de partida a proposta de Miller (1983), Lumpkin e Dess (1996) apresentam o conceito de Orientação Empreendedora com as dimensões, inovação, tomada de risco, proatividade e a partir da citação de Miller (1983, p. 771) "beating competitors to the punch" acrescentaram agressividade competitiva e autonomia, propondo dessa forma para a OE cinco variáveis. Diferentemente de Miller (1983), entretanto, os autores acreditam na multidimensionalidade do construto.

Lumpkin e Dess (1996) definiram a agressividade competitiva como os esforços de uma organização para superar seus adversários no mercado, mantendo uma postura de confronto e autonomia, ou seja, referem-se à forma como as empresas se relacionam e desafiam seus concorrentes, respondendo às tendências e demandas que já existem no mercado.

Autonomia é descrita por Lumpkin e Dess (1996) como a liberdade que os indivíduos têm para exercer sua criatividade e dessa forma gerar ideias promissoras, fornecendo assim um importante impulso para novos empreendimentos. Refere-se à ação independente de um indivíduo ou de uma equipe em dar continuidade a uma ideia ou visão e levá-la a sua conclusão, ou seja, sua liberdade para agir. Os autores argumentam 
que a autonomia é uma dimensão essencial de uma OE. Em um contexto organizacional isso se reflete nas medidas tomadas pelo gestor a fim de suprimir dificuldades organizacionais.

A partir dos trabalhos de Miller (1983) e Lumpkin e Dess (1996) tem-se um arcabouço teórico na literatura sobre $\mathrm{OE}$ dividido em dois posicionamentos distintos. Para Covin e Wales (2011), os pesquisadores possuem liberdade para escolher à medida que melhor serve para seus propósitos, reconhecendo os modelos unidimensional ou multidimensional.

Ambos os autores são amplamente reconhecidos na literatura, entretanto Wales, Gupta e Mousa (2011) fizeram um levantamento na literatura de OE e apontam que $80 \%$ dos artigos empíricos que os autores analisaram adotam o modelo proposto por Miller (1983) considerando a OE como um constructo unidimensional que utiliza as variáveis, inovação, tomada de risco e proatividade. Corroborando este estudo, Kraus et al. (2012) asseveram que a maioria dos estudos têm utilizado combinadamente a tomada de risco, inovação e proatividade para capturar a OE.

\section{METODOLOGIA}

O método escolhido foi o quantitativo. Os procedimentos foram divididos em duas etapas; para a primeira etapa foi realizado o levantamento da literatura existente, foram utilizadas as bases de dados nacionais e internacionais como SPELL, EBSCO, SCOPUS e SUPrimo, que geraram um total de 374 artigos alinhados com a pesquisa. A partir deste levantamento optou-se pela definição do modelo teórico de Orientação Empreendedora de Miller (1983). O questionário utilizado foi operacionalizado por Covin e Slevin (1989). Com o questionário definido foi feita sua tradução do Inglês para o Português e como um passo seguinte foi realizada a tradução reversa para a língua original. $\mathrm{Na}$ sequência foi feita a definição da população e amostra que farão parte deste trabalho.

A partir de então pôde-se dar início à coleta de dados, para esta etapa foi encaminhado o questionário estruturado com o auxílio do Google Docs para a Associação Brasileira de Empreendedores nos EUA. A definição da população desta pesquisa deu-se de forma intencional por acessibilidade. A população compreendeu 3.657 empreendedores cadastrados na Associação Brasileira de Empreendedores nos EUA. Foi estabelecido contato com o grupo de empreendedores e obtivemos 107 respostas, compondo assim nossa amostra.

O instrumento da presente pesquisa é composto pelo questionário de Orientação Empreendedora do modelo proposto por Miller (1983) e que posteriormente foi aperfeiçoado por Covin e Slevin (1989). O construto é composto por três dimensões, são elas, proatividade, inovação e tomada de risco. Este questionário é composto por 9 perguntas em uma escala de diferencial semântico tipo Likert de 7 pontos

Foram acrescidas ao instrumento de pesquisa perguntas como o número de funcionários que a empresa possui a fim de se verificar o porte, o sexo do empreendedor, a idade e o Estado de origem no Brasil. Este instrumento terá um total de 14 perguntas que serão aplicadas aos empreendedores de empresas de pequeno porte, delimitadas por critério de número de funcionários. 
A aplicação ocorreu de forma eletrônica por e-mail com o auxílio do Google Docs. Todos os instrumentos preenchidos foram organizados em uma planilha eletrônica Excel ${ }^{\circledR}$, para que fosse feito o pré-tratamento dos dados.

$\mathrm{Na}$ análise dos dados apresentou-se a descrição dos dados coletados, a composição da amostra em percentual dos setores analisados, o número de funcionários por empresa, o gênero do respondente, sua idade, o tempo de residência nos Estados Unidos. Na sequência utilizou-se a modelagem de equação estrutural, utilizado o software Smart PLS, para poder verificar e avaliar a carga dos fatores, as medidas de adequação das amostras, o alfa de Cronbach, as correlações das variáveis, e dos constructos (HAIR JR. et al., 2005).

\section{ANÁLISE DOS DADOS E DISCUSSÃO}

A amostra foi composta por 107 empresários de origem brasileira estabelecidos nos Estados Unidos. Quanto ao segmento trabalhado, a partir das respostas verificou-se que $75,7 \%$ das empresas são de prestação de serviços, $18,7 \%$ de comércio e $5,6 \%$ são de indústria.

Para caracterizar o porte da empresa tomou-se como referência o padrão americano, em que empresas com menos de 500 funcionários são caracterizadas como pequenas. Quanto ao número de funcionários identificou-se que a maior empresa estudada possui 60 funcionários. A maior parte de nossa amostra é composta por empresas que possuem até 10 colaboradores, o que corresponde a $84 \%$ da amostra. Ainda cabe ressaltar que 53,3\% das empresas possuem até 2 funcionários. Quanto ao gênero dos empreendedores brasileiros nos Estados Unidos é composto majoritariamente por muIheres, que representam $77,6 \%$, enquanto os homens representam 20,6\%. Desta amostra $1,9 \%$ se declararam LGBT (lésbicas, gays, bissexuais e transexuais).

No que se refere à idade dos empreendedores, verificou-se que no intervalo da amostra de 0 a 19 anos há $0,9 \%$, de 20 a 29 anos há $21,5 \%$, de 30 a 39 anos há $36,4 \%$, de 40 a 49 anos há $26,2 \%$, de 50 a 59 anos há $13,1 \%$, de 60 a 69 anos há 1,9\% e não foram encontrados empreendedores com mais de 70 anos. No que se refere ao tempo de residência nos Estados Unidos, 57\% dos empresários brasileiros residem no país há mais de 10 anos, $11,2 \%$ estão no país há mais de 20 anos, 18,7\% estão no país de 16 a 20 anos, $27,1 \%$ de 11 a 15 anos, 19,6\% de 6 a 10 anos, 10,3\% de 2 a 5 anos e 13,1 \% há menos de 2 anos.

Dando prosseguimento à análise dos dados, utilizou-se modelagem de equações estruturais SEM (Structural Equation Modeling) que para Hair Jr et. al. (2014) envolve a aplicação de métodos estatísticos que analisam múltiplas variáveis simultaneamente. Para que seja válido o modelo, deve-se cumprir alguns requisitos, inicialmente deve-se estimar o modelo no PLS com todas as questões originais de cada construto; na sequência deve-se avaliar quais relacionamentos possuíram um bom ajuste, indicadores com carga inferior a 0,5 devem ser excluídos e o modelo rodado novamente. A condição ideal é que os relacionamentos possuam cargas maiores que 0,7 e AVE (Average Variance Extracted) superior a 0,5. Na sequência avalia-se a validade discriminante e a confiabilidade; vencida esta etapa pode-se verificar os coeficientes estruturais e $R^{2}$ para então chegar ao modelo final (HAIR JR. et al., 2014; SILVA, 2015). 
A primeira etapa consiste em estimar o modelo no PLS com todas as questões originais de cada construto. O modelo apresenta a OE com três dimensões, tomada de risco, inovação e proatividade. A dimensão tomada de risco apresenta um coeficiente de caminho com o construto $\mathrm{OE}$ de 0,853 , a dimensão proatividade se relaciona com a OE em 0,881 e a dimensão inovação gerou um escore de 0,759 . Todas as dimensões apresentaram um bom relacionamento com o construto. De acordo com Hair Jr. et al. (2014), valores próximos a 1 indicam um bom ajuste do modelo e valores inferiores a 0,7 devem ser excluídos e o modelo deve ser rodado novamente. Como os valores de relacionamento das dimensões com seu construto foram superiores a 0,7 , não houve a necessidade de exclusão, criando assim uma condição ideal.

O passo seguinte foi a avaliação da Variância Média Extraída - AVE (Average Variance Extracted). Na dimensão tomada de risco o valor da AVE é de 0,76, para a dimensão proatividade o valor da AVE é de 0,62 e para inovação é de 0,79. Estes valores de acordo com Hair Jr. et al., (2014) devem ser superiores a 0,5 para que haja uma condição ideal do modelo. Foi calculado o alfa de Cronbach, para avaliar a fiabilidade do questionário. Para a dimensão tomada de risco, o valor do alfa de Cronbach foi de 0,84 , para proatividade foi gerado um escore de 0,70 e para inovação o valor resultante é de 0,6. Para Babbie (1992), os escores gerados pela tomada de risco e proatividade representam uma classificação alta, enquanto o escore oriundo da inovação representa uma classificação moderada.

Como última etapa avaliou-se os coeficientes estruturais. Para a dimensão tomada de risco o $\mathrm{R}^{2}$ resultou no valor de 0,728 , que Hair Jr. et al. (2014) avaliam como moderado. Para proatividade o escore gerado pelo $R^{2}$ foi de 0,776 , que de acordo com Hair Jr. et al. (2014) pode ser considerado substancial. Para inovação o valor resultante do $R^{2}$ é de 0,576 que para Hair Jr. et al. (2014) pode ser considerado moderado. A partir dos resultados oriundos das análises da OE infere-se que o relacionamento entre estas três dimensões para com seu construto na amostra pesquisada mostrou-se satisfatório e revela-se adequado. Esses relacionamentos e os valores resultantes dos cálculos podem ser observados de forma sumária na Figura 1.

Figura 1 - Cargas para o modelo da orientação empreendedora

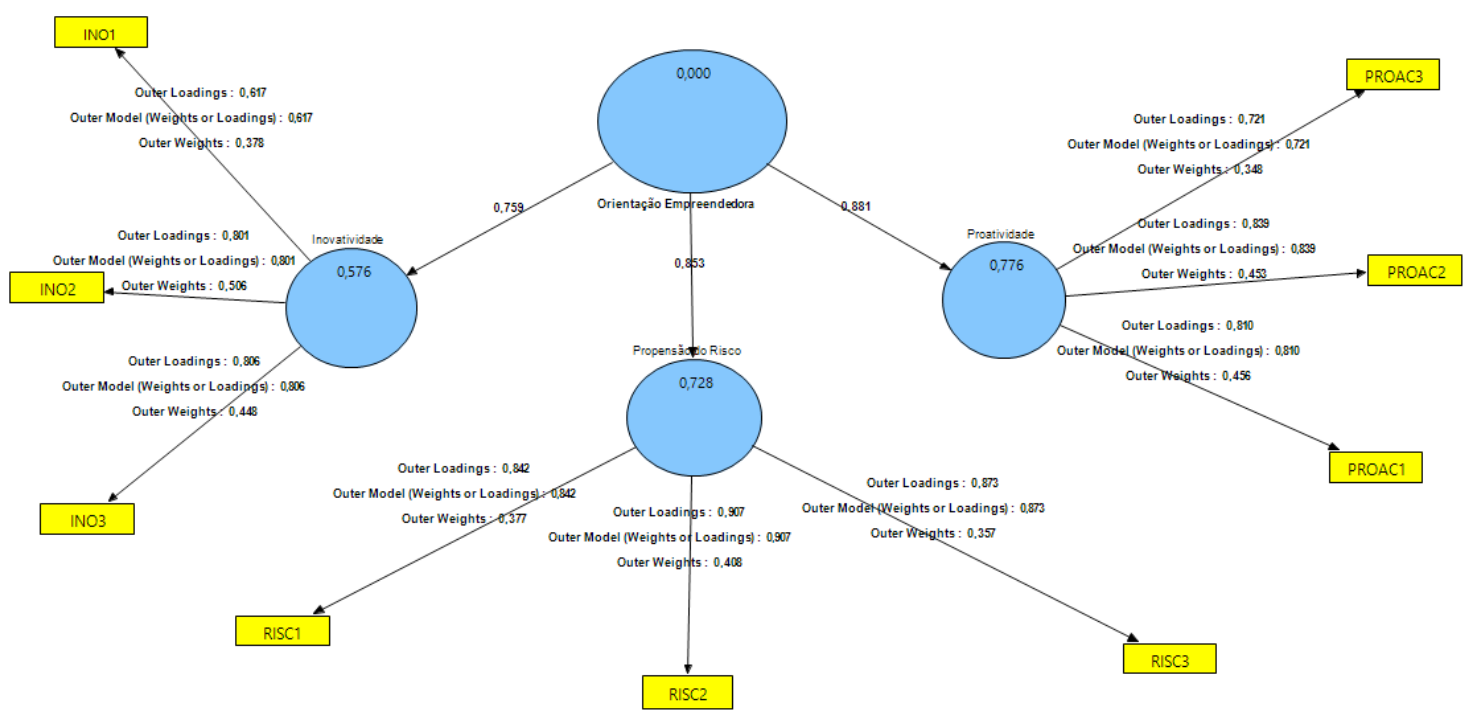

Fonte: Dados da pesquisa. 


\section{CONSIDERAÇÕES FINAIS}

Quanto maior o nível das dimensões de inovação, tomada de risco e proatividade na organização, mais o negócio se torna empreendedor (EGGERS; HANSEN; DAVIS, 2012). A orientação empreendedora aparece quando a organização envolve ações estratégicas, observáveis e especificamente - inovação, proatividade e tomada de riscos - e se essas ações ocorrem com alguma consistência (ANDERSON; ESHIMA, 2013).

A capacidade de inovação de uma organização envolve o uso de uma nova tecnologia em novos produtos ou serviços (MILLER, 1983). Para o alcance do primeiro objetivo específico, que se refere à identificação do grau de inovação nas empresas pesquisadas, obteve-se valores dentro das recomendações de Hair Jr. et al. (2014) para a dimensão inovação, o valor para o coeficiente de caminho foi de 0,76 , esse valor representa um ajuste de $76 \%$ da dimensão com o construto. $O$ valor do $R^{2}$ foi de 0,56 , esse índice se apresenta superior a 0,5 conforme recomendado pela literatura. Também se avaliou a Variância Média Extraída - AVE (Average Variance Extracted), esse valor foi de 0,79 , criando uma condição ideal do modelo. Foi calculado o alfa de Cronbrach para avaliar a fiabilidade do questionário e resultou no valor de 0,6.

O segundo objetivo específico foi alcançado quando se verificou os valores para a dimensão proatividade, que no entendimento de Lumpkin e Dess (1996) refere-se à forma como a empresa se relaciona com o mercado, agindo oportunamente a fim de moldar o ambiente, influenciando tendências ou criando demandas e agindo com perspicácia para aproveitar as oportunidades. Os valores encontrados neste trabalho apresentam um coeficiente de caminho com o escore de 0,88 . Este valor representa um ajuste de $88 \%$ da dimensão proatividade com o construto da Orientação Empreendedora. $O$ valor do $\mathrm{R}^{2}$ foi de 0,78 , o AVE calculado foi de 0,62 , o que representa uma condição ideal do modelo e para avaliar a fiabilidade do questionário foi verificado o alfa de Cronbach, que resultou em 0,6.

O terceiro objetivo específico foi atingido ao calcular os valores para a dimensão tomada de risco. Para Khandwalla (1976), o risco está associado à forma como os gestores interagem, agressivamente ou não, com seu ambiente. Nesta pesquisa foram encontrados os seguintes valores: o coeficiente de caminho gerou um escore de 0,85 , este valor representa um ajuste de $85 \%$ nesta dimensão com o construto da Orientação Empreendedora. $\mathrm{O}$ valor do $\mathrm{R}^{2}$ foi de 0,73 , o valor do AVE foi de 0,76, o que representa uma condição ideal do modelo e para avaliar a fiabilidade do questionário foi verificado o alfa de Cronbach, que resultou em 0,84.

Com os objetivos específicos alcançados, voltamos ao objetivo geral que pretende identificar o grau de Orientação Empreendedora (OE) existente nos pequenos empresários brasileiros que atuam nos EUA. Identificou-se a partir dos dados da pesquisa que a amostra estudada possui as características propostas por Miller (1983) nas seguintes proporções: $56 \%$ para inovação, $78 \%$ para proatividade e $73 \%$ para tomada de risco, 0 que caracteriza os brasileiros que imigraram para os EUA que possuem comportamento altamente proativo, com um forte comprometimento na tomada de risco e apostam medianamente nas inovações. 
A partir dos dados resultantes desta pesquisa observou-se que o modelo de Orientação Empreendedora proposto por Miller (1983) mostra-se elevado para a amostra de pequenos empreendedores brasileiros que atuam nos EUA.

Como propostas para futuras pesquisas sugere-se que este trabalho seja reaplicado com uma população brasileira que imigrou para diferentes países a fim de vislumbrar semelhanças e diferenças nas amostras estudadas. Sugere-se também que seja feita uma análise dos fatores motivacionais que levam os empreendedores a saírem de seu país de origem para empreender em um país diferente.

\section{REFERÊNCIAS}

ANDERSON, B. S.; ESHIMA Y. The influence of firm age and intangible resources on the relationship between entrepreneurial orientation and firm growth among Japanese SMEs. Journal of Business Venturing, v. 28, p. 413-429, 2013.

AVLONITIS, G. J.; SALAVOU, H.E. Entrepreneurial orientation of SMEs, product innovativeness, and performance. Journal of Business Research, v. 60, p. 566-575, 2007.

BABBIE, E. The practice of social research. California: Wardsworth Publishing Company. 1992.

BAIRD, I. S.; THOMAS, H. Toward a Contingency Model of Strategic Risk Taking. Academy of Management Review, v. 10, n. 2, p. 230-243, 1985.

COVIN, J. G.; WALES, W. J. The Measurement of Entrepreneurial Orientation. Entrepreneurship Theory and Practice, volume 36, issue 4, p. 677-702, 2011.

COVIN, J. G.; SLEVIN, D. P. Strategic management of small firms in hostile and benign environments. Strategic Management Journal, v. 10, p. 75-87, 1989.

EGGERS, F.; HANSEN, D. J.; DAVIS, A. E. Examining the relationship between customer and entrepreneurial orientation on nascent firms' marketing strategy. Int Entrep Manag J., v. 8, p. 203-222, 2012.

FILLIS, I. Small firms internationalisation: an investigate survey an future research directions. Management Decisions, v. 39, n. 9, 767-783, 2001.

GIBSON, S.; HARRIS, M. L.; SADIGHIAN, F. Investgating the Entrepreneurial Attitudes of Armenian Immigrants. The Coastal Business Journal, v. 10, n. 1, 2011.

GLOBAL ENTREPRENEURSHIP MONITOR (GEM). 2016. Disponível em: http://www.gemconsortium.org/ country-profile/46. Acesso em: 20 out. 2017.

HAIR JR., Joseph F. et al. A Primer on Partial Least Squares Structural Equation Modeling (PLS-SEM). LoS Angeles: Sage, 2014. 295p.

JAUCH, L. R.; GLUECK W. F. Business policy and strategic management. New York: McGraw-Hill, 1988 KHANDWALLA, P. N. The Design of Effective Top Management Style. Vikalpa, v. 1, n. 2, april 1976.

KRAUS, S. et al. Entrepreneurial orientation and the business performance of SMEs: a quantitative study from the Netherlands. Rev Manag Sci., v. 6, p. 161-182, 2012.

LUMPKIN, G. T.; DESS, G. G. Clarifying the Entrepreneurial Orientation Construct and Linking It to Performance. The Academy of Management Review, v. 21, n. 1, p. 135-172, 1996.

MARGOLIS, M. L. Transnationalism and Popular Culture: The Case of Brazilian Immigrants in the United States. The Journal of Popular Culture, v. 29, n. 1, p. 29-41, 1995.

MERZ, G. R.; SAUBER M. H. Profiles of managerial activities in small firms. Strategic Management Journal, V. 16, p. 551-564, 1995.

MILLER, D. Revisited: A Reflection on EO Research and Some Suggestions for the Future. Entrepreneurship Theory and Practice, p. 873-894, 2011. DOI: 10.1111/j.1540-6520.2011.00457.x.

MILLER, D. The Correlates of Entrepreneurship in Three Types of Firms. Management Science, v. 29, n. 7, p. 770-791, 1983.

MILLER, D.; FRIESEN, P. H. Innovation in Conservative and Entrepreneurial Firms: Two Models of Strategic Momentum. Strategic Management Journal, v. 3, p. 1-25, 1982.

NINIS, A. B. Os pequenos empreendedores no mercado de trabalho. In: BRASIL. Vozes da Nova Classe Média, Presidência da República, Secretaria de Assuntos Estratégicos, caderno 3, p. 27-51, 2013.

PEARCE, J. A.; FRITZ, D. A.; DAVIS, P. S. Entrepreneurial Orientation and the Performance of Religious Congregations as Predicted by Rational Choice Theory. Entrepreneurship Theory and Practice, v. 34, Issue 1, p. 219-248, january 2010. 
SANDERS, J. M.; NEE, V. Immigrant self-employment: the family as social capital and the value of human capital. American Sociological Review, v. 61, p. 231-249, 1996.

SCHUMPETER, J. A. Teorie der wirtschaflichen Entwicklung. Leipzig: Verlag Von Duncker \& Humblot, 1911. (Tradução Maria Sílvia Possas. Teoria do desenvolvimento econômico. Uma investigação sobre lucros, capital, crédito, juro e o ciclo econômico. São Paulo: Nova Cultural, 1997).

SILVA, D. da. Modelagem de equações estruturais usando o SmartPLS. 17 abr. 2015. 290 p. (Notas de aula).

SLEVIN, D. P. TERJESEN, S. A. Entrepreneurial Orientation: Reviewing Three Papers and Implications for Further Theoretical and Methodological Development. Entrepreneurship Theory and Practice. p. 973-987. September, 2011. DOI: 10.1111/j.1540-6520.2011.00483.x1042-2587.

SOININEN, J. et al. Entrepreneurial orientation: Growth and profitability of Finnish small- and medium-sized enterprises. Int. J. Production Economics, v. 140, p. 614-621, 2012.

SUNDQVIST, S.; KUIVALAINEN, O.; CADOGAN, J. W. Contingency Factors in International Entrepreneurial Orientation - Performance Relations of Firms with Different Levels of Internationalization. Firm-Level Internationalization, Regionalism and Globalization: Strategy, Performance and Institutional Change. Editado por BERRILL, J.; HUTSON, E.; SINKOVICS, R. London: Palgrave Macmillan, 2011.

VOSS, Z. G.; VOSS, G. B.; MOORMAN, C. An empirical examination of the complex relationships between entrepreneurial orientation and stakeholder support. European Journal of Marketing, v. 39, n. 9/10, 2005. WALES, W. J.; GUPTA, V. K.; MOUSA, F-T. Empirical research on entrepreneurial orientation: An assessment and suggestions for future research. International Small Business Journal, v. 31, n. 4, p. 357-383, 2011.

WIKLUND, J.; SHEPHERD, D. A. Entrepreneurial orientation and small business performance: a configurational approach .Journal of Business Venturing, v. 20, p. 71-91, 2005.

WIKLUND, J.; SHEPHERD, D. A. Portfolio Entrepreneurship: Habitual and Novice Founders, New Entry, and Mode of Organizing. Entrepreneurship theory and Practice, p. 701-725, july 2008. 